\title{
(2) OPEN ACCESS \\ Prevalence of smoking in adults with spinal cord stimulators: a systematic review and meta-analysis
}

\author{
W Michael Hooten @i , ${ }^{1}$ Rajat N Moman, ${ }^{1}$ Jodie Dvorkin, ${ }^{2}$ E Morgan Pollard, \\ Robalee Wonderman, ${ }^{1}$ M Hassan Murad $^{3}$
}

Additional material is published online only. To view please visit the journal online (http://dx.doi.org/10.1136/ rapm-2019-100996).

${ }^{1}$ Anesthesiology and Perioperative Medicine, Mayo Clinic Rochester, Rochester, Minnesota, USA

Institute for Clinical Systems Improvement, Bloomington, Minnesota, USA

${ }^{3}$ Preventive Medicine, Mayo Clinic Rochester, Rochester,

Minnesota, USA

\section{Correspondence to} Dr W Michael Hooten, Anesthesiology, Mayo Clinic, Rochester, MN 55905, USA; hooten.william@mayo.edu

The study findings have not been previously presented at a scientific meeting.

Received 14 September 2019 Revised 13 November 2019 Accepted 4 December 2019 Published Online First 28 January 2020

\begin{abstract}
Background Smoking adversely impacts pain-related outcomes of spinal cord stimulation (SCS). However, the proportion of SCS patients at risk of worse outcomes is limited by an incomplete knowledge of smoking prevalence in this population. Thus, the primary aim of this systematic review is to determine the prevalence of smoking in adults with chronic pain treated with SCS. Methods A comprehensive search of databases from 1 January 1980 to 3 January 2019 was conducted. Eligible study designs included (1) randomized trials; (2) prospective and retrospective cohort studies; and (3) cross-sectional studies. The risk of bias was assessed using a tool specifically developed for prevalence studies. A total of 1619 records were screened, 19 studies met inclusion criteria, and the total number of participants was 10838.

Results Thirteen studies had low or moderate risk of bias, and six had a high risk of bias. All 19 studies reported smoking status and the pooled prevalence was $38 \%$ (95\% Cl 30\% to 47\%). The pooled prevalence in 6 studies of peripheral vascular diseases was $56 \%$ (95\% $\mathrm{Cl} 42 \%$ to $69 \%$ ), the pooled prevalence of smoking in 11 studies of lumbar spine diagnoses was $28 \%$ (95\% Cl $20 \%$ to $36 \%$ ) and the pooled prevalence in 2 studies of refractory angina was $44 \%(95 \% \mathrm{Cl} 31 \%$ to $58 \%)$.

Conclusions The estimated prevalence of smoking in SCS patients is 2.5 times greater than the general population. Future research should focus on development, testing and deployment of tailored smoking cessation treatments for SCS patients.
\end{abstract}

\section{INTRODUCTION}

Over the past 25 years, technological and surgical advances have revolutionized the use of spinal cord stimulation (SCS) for a broad range of chronic pain conditions $^{1}$; however, not all patients experience optimal long-term benefits. One recently identified subgroup that may be at risk of poor outcomes are smokers treated with SCS. In a retrospective study that involved 213 patients with chronic spinerelated pain treated with SCS, smokers reported greater pain intensity and consumed greater quantities of opioids compared with nonsmokers at 1 year following SCS implant. ${ }^{2}$ More specifically, the pain scores of smokers were $37 \%$ greater compared with never smokers, and the median morphine equivalent dose of smokers was $40 \mathrm{mg}$ compared with 23 $\mathrm{mg}$ in never smokers. ${ }^{23}$ This is critically important because SCS is one of the few treatments associated with sustained and durable pain relief, and low risk of adverse effects. ${ }^{1}$

The proportion of SCS patients impacted by the adverse influence of smoking is limited, in part, by an incomplete knowledge of smoking prevalence in adults with SCS. Thus, the primary aim of this systematic review is to determine the prevalence of smoking in adults with chronic pain treated with SCS. A secondary aim includes determining the prevalence of smoking in adults with chronic pain based on the indication for SCS including lumbar spine diagnoses, ischemic pain related to peripheral vascular diseases and refractory angina.

\section{METHODS}

\section{Study protocol}

Preferred Reporting Items for Systematic Reviews and Meta-Analyses guidelines ${ }^{4}$ were followed. An a priori protocol was followed. The trial was registered in the PROSPERO database (CRD42018117004). ${ }^{5}$

\section{Search strategy}

A comprehensive search of databases from 1 January 1980 to 3 January 2019 was conducted. The databases included MEDLINE Epub Ahead of Print, Medline In-Process and Other Non-Indexed Citations, MEDLINE, EMBASE and Scopus. The search strategy was designed and conducted by a medical reference librarian with input from the principal investigator. No language restrictions were employed. Controlled vocabulary supplemented with keywords was used to search for studies on the prevalence of smoking in patients with SCSs. The search strategy is provided in online supplementary appendix A.

\section{Study selection process}

Study inclusion criteria included (1) randomized, cross-over and parallel-designed clinical trials; (2) prospective and retrospective cohort studies; (3) cross-sectional design; (4) age 18 years or older; (5) publication years 1980 to present; (6) any publication language; (7) studies of patients with SCSs and (8) study results that included data on smoking prevalence. Exclusion criteria included (1) studies that involved patients without chronic pain and (2) studies of adults with acute or subacute pain.

In the first phase, two independent pairs of reviewers screened all titles and abstracts identified by our search strategy. In the second phase, the two pairs of independent reviewers screened the bibliography of each full-text article to identify additional relevant citations for inclusion. In the third phase, 
all full-text articles were screened for inclusion and exclusion criteria and the reason for exclusion of each full text was noted.

\section{Data extraction}

Data were extracted by four independent reviewers using a templated electronic database.

Based on the a priori protocol, abstracted data included study design, number of subjects, indication for SCS placement, demographics (mean age, percent female sex) and prevalence of smoking. Smoking status was categorized as current smoker, non-smoker or former smoker. In individual studies, participants described as 'smokers', 'history of smoking' and 'tobacco use' were considered to be current smokers. At least two attempts were made to contact investigators for incompletely reported outcomes.

\section{Risk of bias assessment}

The risk of bias in the included studies was assessed by two independent reviewers using a tool specifically developed to assess risk in prevalence studies. ${ }^{6}$ A summary risk of bias was reported for each manuscript included in the study. Reviewer discrepancy was resolved by consensus or by a third reviewer.

\section{Evidence synthesis}

For each trial, the prevalence and SE were recorded. If the SE was not reported, it was calculated from the prevalence data. A random-effects model was used and results were reported with
95\% CIs. Statistical analyses were performed using Stata V.15 (StataCorp. 2017. Stata Statistical Software: Release 15).

\section{RESULTS}

\section{Characteristics of included studies}

A flow diagram of the study selection process is depicted in figure 1. A total of 19 studies met inclusion criteria (online supplementary file 1). ${ }^{2-24}$ All 19 studies reported current smoking and 2 studies ${ }^{2} 21$ reported former smoking. Three studies were randomized controlled trials (RCTs), ${ }^{13} 2124$ six studies were prospective cohort studies, ${ }^{7-9} 111516$ nine studies were retrospective cohort studies ${ }^{2} 10121417-2023$ and one study was a cross-sectional study. ${ }^{22}$ Demographic and smoking information were available for 10838 SCS patients. The general indication for SCS placement in 11 studies was lumbar spine diagnoses including chronic low back pain, radiculopathy, failed back surgery syndrome, degenerative disc disease and spinal stenosis. $^{28-11141719202223}$ The indication for SCS placement in six studies was peripheral vascular diseases and, in all studies, pain was an inclusion criterion or a key outcome measure. ${ }^{712} 16182124$ The indication for SCS in two studies was refractory angina. ${ }^{13} 15$

\section{Risk of bias and level of certainty}

The summary results of the risk of bias evaluation are listed in online supplementary file. Thirteen studies were found to have a low or moderate risk of bias ${ }^{2-12} 141^{17-2124}$ and six were found to

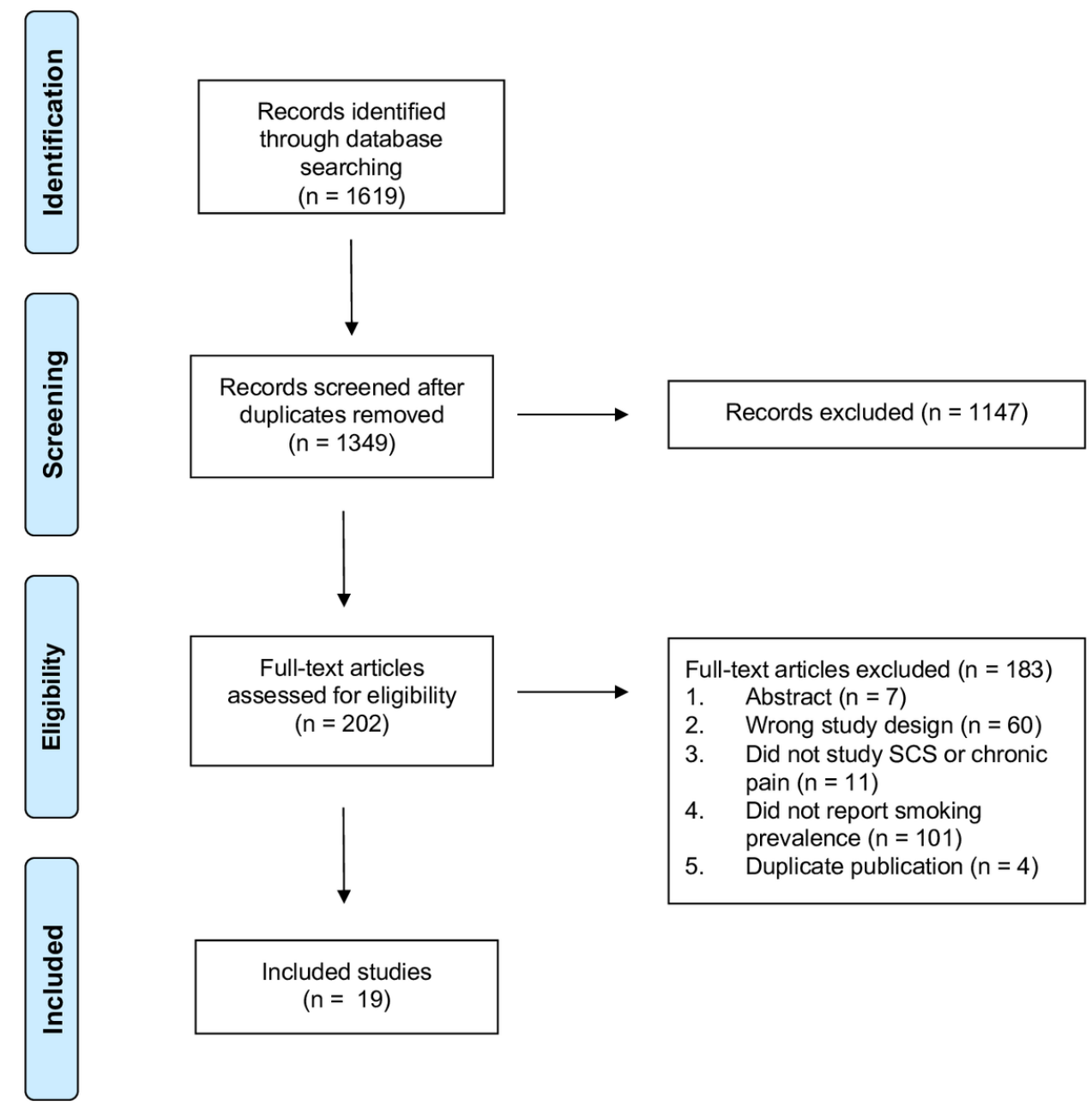

Figure 1 Preferred Reporting items for Systematic Reviews and Meta-Analyses flow chart of the study selection process. ${ }^{3}$ 


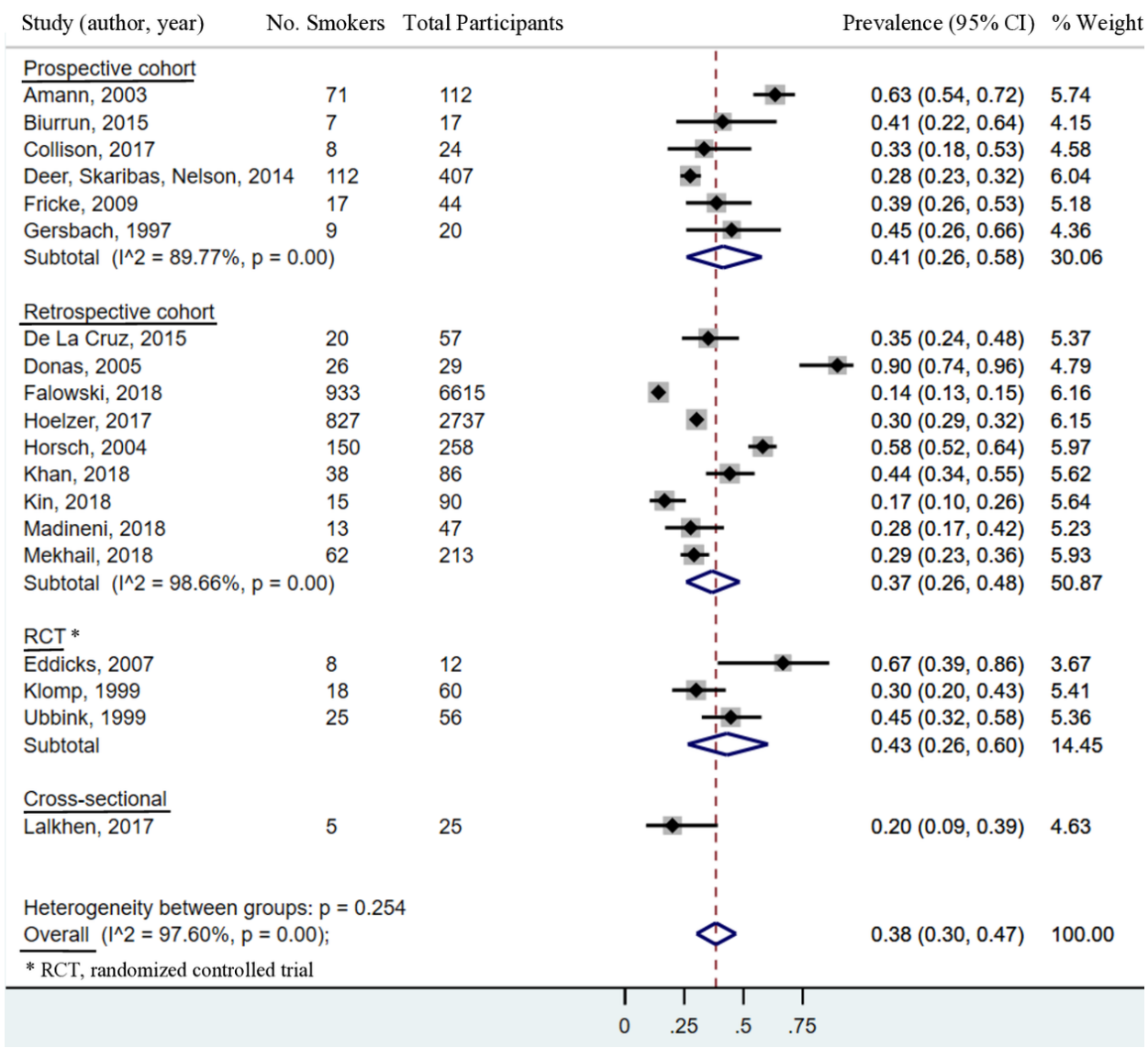

Figure 2 Prevalence of smoking in adults treated with spinal cord stimulation based on study design.

have a high risk of bias. ${ }^{71315162223}$ The majority of differences in the risk of bias centered around the first two questions: (1) Is the study population a close representation of the national population? and (2) Is the sampling frame a true or close representation of the target population?

\section{Prevalence of smoking based on study design}

All 19 studies ( $n=10838$ patients) reported current smoking and the prevalence ranged from $14 \%$ to $90 \%$ (figure 2 ). The pooled prevalence of smoking in these studies was 38\% $(95 \% \mathrm{CI}$ $30 \%$ to $47 \%)$ with high heterogeneity $\left(\mathrm{I}^{2}=97.6 \%\right)$. When one large study that used administrative claims data was excluded from the meta-analysis, ${ }^{14}$ the pooled prevalence increased to $41 \%$ (95\% CI 33\% to $48 \%$ ).

The prevalence of smoking in six prospective cohort studies ranged from $28 \%$ to $63 \%$ (figure 2). The pooled prevalence of smoking in these studies was $41 \%$ (95\% CI $26 \%$ to $58 \%$ ) with high heterogeneity $\left(\mathrm{I}^{2}=89.8 \%\right)$.

The prevalence of smoking in nine retrospective studies ranged from $14 \%$ to $90 \%$ (figure 2). The pooled prevalence in these studies was $37 \%$ (95\% CI 26\% to 48\%) with high heterogeneity $\left(\mathrm{I}^{2}=98.7 \%\right)$.

The prevalence of smoking in three RCTs ranged from $30 \%$ to $67 \%$ (figure 2 ). The pooled prevalence of smoking in these studies was $43 \%$ (95\% CI 26\% to 60\%). In meta-analyses with three or fewer studies, the $\mathrm{I}^{2}$ estimation becomes unreliable; thus, it was not calculated.

In a single cross-sectional study, the prevalence of smoking was 20\% (figure 2).

\section{Prevalence of smoking based on indication for SCS}

The indication for SCS placement was categorized into three groups including (1) lumbar spine diagnoses ( $\mathrm{n}=11$ studies); (2) peripheral vascular diseases ( $n=6$ studies) and (3) refractory angina ( $\mathrm{n}=2$ studies).

The prevalence of smoking in six studies of patients with peripheral vascular diseases ranged from 30\% to $90 \%$ (figure 3 ). The pooled prevalence of smoking in these studies was $56 \%$ (95\% CI $42 \%$ to $69 \%$ ) with high heterogeneity $\left(\mathrm{I}^{2}=87.6 \%\right)$.

The prevalence of smoking in 11 studies of patients with lumbar spine diagnoses ranged from $14 \%$ to $44 \%$ (figure 3 ). The pooled prevalence of smoking in these studies was $28 \%(95 \% \mathrm{CI}$ $20 \%$ to $36 \%)$ with high heterogeneity $\left(\mathrm{I}^{2}=97.3 \%\right)$.

The prevalence of smoking in two studies of patients with refractory angina ranged from $39 \%$ to $67 \%$ (figure 3). The pooled prevalence of current smoking in these studies was $44 \%$ (95\% CI $31 \%$ to $58 \%$ ). In meta-analyses with three or fewer studies, the $\mathrm{I}^{2}$ estimation becomes unreliable; thus, it was not calculated.

\section{DISCUSSION}

The main finding of this systematic review and meta-analysis was that the pooled prevalence of smoking in adults with chronic pain treated with SCS was 38\%. When the meta-analysis was restricted to prospective studies, the prevalence increased to $41 \%$. The greatest prevalence was observed among adults with peripheral vascular diseases (56\%) and refractory angina (44\%) compared with individuals with lumbar spine diagnoses (28\%).

The estimated prevalence of smoking in the general population has declined over the past 20 years to approximately $15 \%$. $^{25}$ 


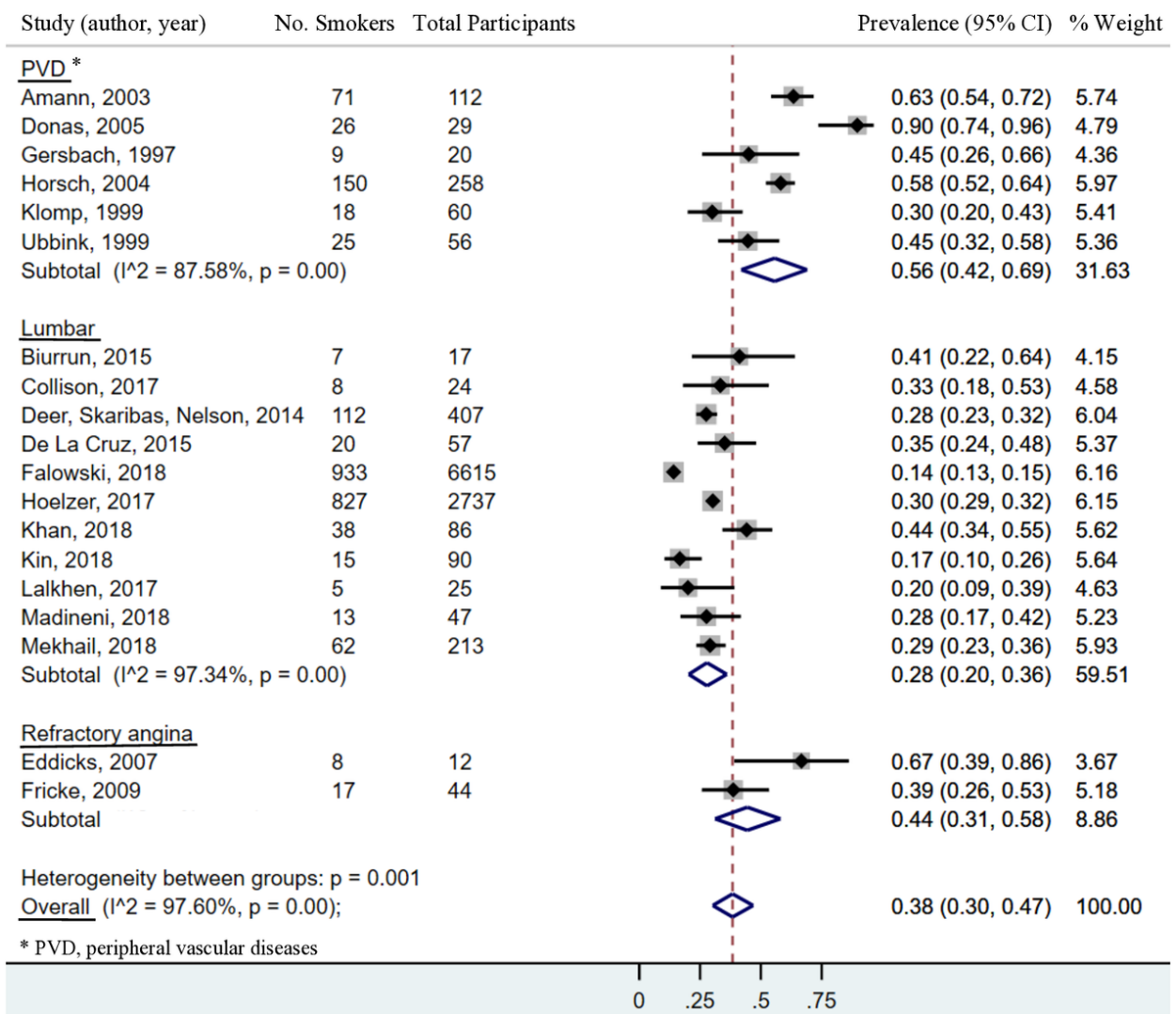

Figure 3 Prevalence of smoking based on the indication for spinal cord stimulation.

However, the prevalence of smoking among individuals with chronic pain remains greater than the general population. ${ }^{26-30}$ More specifically, in a retrospective study that involved 5350 adults with chronic pain admitted to an outpatient functional restoration programme from 1998 to 2012, the prevalence of smoking during the 15 -year study period was $23.5 \% .^{30}$ In this previous study, the overall prevalence of smoking in patients with commonly occurring pain conditions including fibromyalgia, low back pain and headache was 25.2\%, 22.8\% and $21.2 \%$, respectively. ${ }^{30}$ In comparison, the observations from this meta-analysis suggest that the prevalence of smoking in adults treated with SCS is approximately 1.6 times greater than nonSCS treated adults with chronic pain and 2.5 times greater than the general population.

The high prevalence of smoking in adults with chronic pain could be due, in part, to clinical factors unique to this patient population. Adult smokers with chronic pain report greater pain intensity, ${ }^{3132}$ which is partly mediated by greater levels of depression $^{33}$ and positively associated with the desire to smoke. ${ }^{34}$ Smokers with chronic pain also experience greater levels of functional impairment ${ }^{35}$ and negative affect, ${ }^{36}{ }^{37}$ are more likely to use opioids, ${ }^{38} 39$ and report that smoking is an important coping strategy for pain and distress. ${ }^{40} 41$ These characteristics could have contributed to the observations reported by Mekhail et al ${ }^{2}$ where smokers treated with SCS reported greater pain scores and consumed greater quantities of opioids compared with nonsmokers.

The results of this study highlight that smoking is a substantial problem in SCS patients which suggests barriers to smoking cessation exist in this population of patients. Although studies suggest that many smokers with chronic pain are willing to consider cessation, it may be difficult for them to quit for several reasons. ${ }^{3542}$ Acute nicotine deprivation for $12-24$ hours is associated with increased pain intensity and alterations in pain perception. ${ }^{43}$ In addition, greater levels of pain-related anxiety are associated with early relapse, ${ }^{45}$ which is particularly important because smokers with chronic pain are less likely to tolerate adverse psychological states. ${ }^{41}$ Thus, smokers with chronic pain, including those treated with SCS, may require tailored interventions that address smoking cessation in the context of chronic pain. $^{42} 46$

This study has limitations including high levels of observed heterogeneity. Although 13 of 19 studies were assessed as being at low to moderate risk of bias, the majority of differences in the risk of bias were related to selection bias in that the study cohorts were not representative of the targeted population. This sources of heterogeneity could have influenced the reported prevalence of smoking. Other potential sources of heterogeneity include variations in how smoking status was assessed in the different study designs. For example, the pooled prevalence of smoking in the retrospective studies was 37\% compared with $41 \%$ in the prospective cohort studies and $43 \%$ in the RCTs. The lower prevalence in the retrospective studies, including the study that used administrative claims data, ${ }^{14}$ could be due to inaccurate or incomplete assessment of smoking status compared with the assessment of smoking in the prospective cohort and RCT designed studies.

In summary, the estimated prevalence of smoking in adults treated with SCS is 2.5 times greater than the general population and 1.6 times greater than non-SCS treated adults with chronic pain. These findings also support the assertion that smoking status should be assessed in future neuromodulation trials. The long-term goal of this area of research is to develop, test, deploy and disseminate effective smoking cessation treatments for adults 
treated with SCS. It is anticipated that the outcomes of future studies will demonstrate the need for preimplant and postimplant smoking cessation interventions in order to effectively mitigate the deleterious effects of smoking on SCS outcomes.

Contributors WMH took part in design of this project, interpretation of data and writing the manuscript. RNM took part in design of this project, acquisition of data, analysis and interpretation of data, and writing the manuscript. JD, EMP and RW took part in acquisition of data. MHM took part in conception and design of this project, and analysis and interpretation of data.

Funding The authors have not declared a specific grant for this research from any funding agency in the public, commercial or not-for-profit sectors.

\section{Competing interests None declared.}

Patient consent for publication Not required.

Provenance and peer review Not commissioned; externally peer reviewed.

Data availability statement All data relevant to the study are included in the article or uploaded as online supplementary information.

Open access This is an open access article distributed in accordance with the Creative Commons Attribution Non Commercial (CC BY-NC 4.0) license, which permits others to distribute, remix, adapt, build upon this work non-commercially, and license their derivative works on different terms, provided the original work is properly cited, an indication of whether changes were made, and the use is noncommercial. See: http://creativecommons.org/licenses/by-nc/4.0/.

\section{ORCID iD}

W Michael Hooten http://orcid.org/0000-0001-5645-6355

\section{REFERENCES}

1 Lamer TJ, Deer TR, Hayek SM. Advanced innovations for pain. Mayo Clin Proc 2016:91:246-58.

2 Mekhail N, Azer G, Saweris Y, et al. The impact of tobacco cigarette smoking on spinal cord stimulation effectiveness in chronic spine-related pain patients. Reg Anesth Pain Med 2018;43:1-75.

3 Hooten WM. The effects of smoking on spinal cord stimulation outcomes: another reason to quit. Reg Anesth Pain Med 2018;43:687-8

4 Moher D, Liberati A, Tetzlaff J, et al. Preferred reporting items for systematic reviews and meta-analyses: the PRISMA statement. BMJ 2009:339:b2535.

5 Booth A, Clarke M, Ghersi D, et al. An international registry of systematic-review protocols. The Lancet 2011:377:108-9.

6 Hoy D, Brooks P, Woolf A, et al. Assessing risk of bias in prevalence studies: modification of an existing tool and evidence of interrater agreement. J Clin Epidemiol 2012;65:934-9

7 Amann W, Berg P, Gersbach P, et al. Spinal cord stimulation in the treatment of non-reconstructable stable critical leg ischaemia: results of the European peripheral vascular disease outcome study (SCS-EPOS). Eur J Vasc Endovasc Surg 2003;26:280-6

8 Biurrun Manresa JA, Sörensen J, Andersen OK, et al. Dynamic changes in nociception and pain perception after spinal cord stimulation in chronic neuropathic pain patients. Clin J Pain 2015;31:1046-53

9 Collison C, Prusik J, Paniccioli S, et al. Prospective study of the use of intraoperative neuromonitoring in determining post-operative energy requirements and physiologic midline in spinal cord stimulation. Neuromodulation 2017;20:575-81.

10 De La Cruz P, Fama C, Roth S, et al. Predictors of spinal cord stimulation success. Neuromodulation 2015;18:599-602. Discussion 602.

11 Deer T, Skaribas I, McJunkin T, et al. Results from the partnership for advancement in neuromodulation registry: a 24-month follow-up. Neuromodulation 2016;19:179-87.

12 Donas KP, Schulte S, Ktenidis K, et al. The role of epidural spinal cord stimulation in the treatment of Buerger's disease. J Vasc Surg 2005;41:830-6.

13 Eddicks S, Maier-Hauff K, Schenk M, et al. Thoracic spinal cord stimulation improves functional status and relieves symptoms in patients with refractory angina pectoris: the first placebo-controlled randomised study. Heart 2007:93:585-90.

14 Falowski SM, Provenzano DA, Xia Y, et al. Spinal cord stimulation infection rate and risk factors: results from a United States payer database. Neuromodulation 2019;22:179-89.

15 Fricke E, Eckert S, Dongas A, et al. Myocardial perfusion after one year of spinal cord stimulation in patients with refractory angina. Nuklearmedizin 2009;48:104-9.

16 Gersbach P, Hasdemir MG, Stevens RD, et al. Discriminative microcirculatory screening of patients with refractory limb ischaemia for dorsal column stimulation. Eur J Vasc Endovasc Surg 1997; 13:464-71

17 Hoelzer BC, Bendel MA, Deer TR, et al. Spinal cord stimulator implant infection rates and risk factors: a multicenter retrospective study. Neuromodulation 2017:20:558-62.
18 Horsch S, Schulte S, Hess S. Spinal cord stimulation in the treatment of peripheral vascular disease: results of a single-center study of 258 patients. Angiology 2004;55:111-8.

19 Khan $\mathrm{H}$, Pilitsis JG, Prusik J, et al. Pain remission at one-year follow-up with spinal cord stimulation. Neuromodulation 2018;21:101-5

20 Kin K, Agari T, Yasuhara T, et al. The factors affecting the difficulty of percutaneous cylindrical electrode placement for spinal cord stimulation. World Neurosurg 2018:113:e391-8.

21 Klomp HM, Spincemaille GHJJ, Steyerberg EW, et al. Spinal-Cord stimulation in critical limb ischaemia: a randomised trial. The Lancet 1999:353:1040-4

22 Lalkhen AG, Chincholkar M, Patel J. Microbiological Evaluation of the Extension Wire and Percutaneous Epidural Lead Anchor Site Following a "2-Stage Cut-Down" Spinal Cord Stimulator Procedure. Pain Pract 2017;17:886-91.

23 Madineni RA, Smith CM, Clark SW, et al. Effect of preoperative opioid dosage on postoperative period after thoracic spinal cord stimulator surgery. Pain Med 2018; 19:693-8.

24 Ubbink DT, Spincemaille GHJ, Prins MH, et al. Microcirculatory investigations to determine the effect of spinal cord stimulation for critical leg ischemia: the Dutch multicenter randomized controlled trial. J Vasc Surg 1999;30:236-44.

25 Agaku IT, King BA, Dube SR. Current cigarette smoking among adults - united states, 2005-2012. MMWR Morb Mortal Wkly Rep 2014;63:29-34.

26 Alkherayf F, Agbi C. Cigarette smoking and chronic low back pain in the adult population. CIM 2009;32:360-7.

27 Fishbain DA, Lewis JE, Bruns D, et al. The prevalence of smokers within chronic pain patients and highest pain levels versus comparison groups. Pain Med 2013;14:403-16

28 Palmer KTet al. Smoking and musculoskeletal disorders: findings from a British national survey. Ann Rheum Dis 2003;62:33-6.

29 Scott SC, Goldberg MS, Mayo NE, et al. The association between cigarette smoking and back pain in adults. Spine 1999;24:1090-8.

30 Orhurhu VJ, Pittelkow TP, Hooten WM. Prevalence of smoking in adults with chronic pain. Tob Induc Dis 2015;13:17.

31 Bakhshaie J, Ditre JW, Langdon KJ, et al. Pain intensity and smoking behavior among treatment seeking smokers. Psychiatry Res 2016;237:67-71.

32 De Vita MJ, Maisto SA, Ansell EB, et al. Pack-Years of tobacco cigarette smoking as a predictor of spontaneous pain reporting and experimental pain reactivity. Exp Clin Psychopharmacol 2019.

33 Hooten MW, Shi Y, Gazelka HM, et al. The effects of depression and smoking on pain severity and opioid use in patients with chronic pain. Pain 2011;152:223-9.

34 Kosiba JD, Zale EL, Ditre JW. Associations between pain intensity and urge to smoke: testing the role of negative affect and pain catastrophizing. Drug Alcohol Depend 2018;187:100-8.

35 Hooten WM, Townsend CO, Bruce BK, et al. Effects of smoking status on immediate treatment outcomes of multidisciplinary pain rehabilitation. Pain Med 2009; 10:347-55

36 Smit T, Peraza N, Garey L, et al. Pain-Related anxiety and smoking processes: the explanatory role of dysphoria. Addict Behav 2019;88:15-22.

37 Paulus DJ, Garey L, Gallagher MW, et al. Pain severity as a predictor of negative affect following a self-guided quit attempt: an ecological momentary assessment study. Am J Drug Alcohol Abuse 2018;44:543-50.

38 Hooten WM, Townsend CO, Bruce BK, et al. Sex differences in characteristics of smokers with chronic pain undergoing multidisciplinary pain rehabilitation. Pain Med 2009;10:1416-25.

39 Hooten WM, Townsend $\mathrm{CO}$, Bruce BK, et al. The effects of smoking status on opioid tapering among patients with chronic pain. Anesth Analg 2009:108:308-15.

40 Hooten WM, Vickers KS, Shi Y, et al. Smoking cessation and chronic pain: patient and pain medicine physician attitudes. Pain Pract 2011;11:552-63.

41 LaRowe LR, Farris SG, Zvolensky MJ, et al. Associations between past-month pain and distress intolerance among daily cigarette smokers. I Stud Alcohol Drugs 2018;79:781-9.

42 Hooten WM, LaRowe LR, Zale EL, et al. Effects of a brief pain and smoking cessation intervention in adults with chronic pain: a randomized controlled trial. Addict Behav 2019;92:173-9.

43 Ditre JW, Zale EL, LaRowe LR, et al. Nicotine deprivation increases pain intensity, neurogenic inflammation, and mechanical hyperalgesia among daily tobacco smokers. J Abnorm Psychol 2018;127:578-89.

44 LaRowe LR, Kosiba JD, Zale EL, et al. Effects of nicotine deprivation on current pain intensity among daily cigarette smokers. Exp Clin Psychopharmacol 2018;26:448-55.

45 LaRowe LR, Langdon KJ, Zvolensky MJ, et al. Pain-Related anxiety as a predictor of early lapse and relapse to cigarette smoking. Exp Clin Psychopharmacol 2017;25:255-64.

46 Hooten WM, Townsend CO, Hays JT, et al. A cognitive behavioral smoking abstinence intervention for adults with chronic pain: a randomized controlled pilot trial. Addict Behav 2014:39:593-9. 